\title{
A spatial regularization method preserving local photometry for Richardson-Lucy restoration ${ }^{\star}$
}

\author{
E. Bratsolis ${ }^{1,2}$ and M. Sigelle ${ }^{1}$ \\ 1 École Nationale Supérieure des Télécommunications, Département de Traitement du Signal et des Images, \\ 46 rue Barrault, 75634 Paris Cedex 13, France \\ 2 Institute of Astronomy and Astrophysics, National Observatory of Athens, PO Box 20048, 11810 Athens, Greece
}

Received 31 August 1999 / Accepted 9 April 2001

\begin{abstract}
In this article we give a simple method of spatial regularization deriving from the Richardson-Lucy $(\mathrm{RL})$ algorithm in order to overcome the problem of noise amplification during the image reconstruction process. It is very important in astronomy to regularize images while controlling their photometric behavior. We propose a new reconstruction method preserving both the global and local image photometric properties. A mathematical presentation is described here. This method was applied to an image of Titan - a satellite of Saturn - acquired in near infrared with the adaptive optics system ADONIS installed at the ESO $3.6 \mathrm{~m}$ telescope in La Silla (Chile). The local photometric results here are compared using contours normalized at the same intensity levels.
\end{abstract}

Key words. techniques: image processing - method: data analysis

\section{Introduction}

The success of the RL algorithm (Richardson 1972; Lucy 1974; Shepp \& Vardi 1982) is that it forces the restored image to be non-negative and to keep global flux (photometry) preserved at each iteration. A well-known side effect of the RL algorithm is that it produces highly unstable solutions, namely with high peaks and deep valleys. Our aim is to modify the RL algorithm in order to regularize it while keeping the positivity constraint and the total photometry.

There are two types of noise (Snyder et al. 1993; Snyder et al. 1995). The first one is not associated with the true signal and comes from backgrounds such as the read-out noise of a detector, which has a Gaussian distribution, or the sky background. The sky background noise can be removed before any other data processing, whereas the read-out noise can be modified into a Poisson distribution by a simple modification of data when its Gaussian distribution is known, provided its variance is large enough (Snyder et al. 1993).

The second type of noise is associated with the signal recording process, and is in our case due to photon Poisson statistics in CCD detectors. A regularization method has

Send offprint requests to: E. Bratsolis,

e-mail: bratsoli@tsi.enst.fr

* Research supported by a Marie Curie Fellowship of the European Community programme MCFI under contract number HPMF-CT-2000-00532. to be applied to remove this type of noise. We assume in the following that read-out and sky background noise have been corrected, so that only Poisson noise will be considered.

The problem of highly unstable solutions such as Gibbs oscillations or ringing was solved for the case of point sources whose locations are assumed to be known. The related algorithm, known as PLUCY (Lucy 1994; Hook \& Lucy 1994; Hook \& Lucy 1995), splits the image lying in the object plane into two channels, one for the point sources and one for the background, and leads to a very good photometric fidelity. The problem now is to study what happens if we have an extended object without point source stars but only noise point sources. The wavelet decomposition proposed by Starck et al. (1994) and Starck \& Murtagh (1994) applied to extended objects, like a simulated elliptical galaxy with Poisson and Gaussian (read-out) noise (Murtagh et al. 1995), yields a very good photometric fidelity between the "true image" and the regularized RL restoration. The same wavelet decomposition has been used by Núñez \& Lacer (1998) by using a FMAPE algorithm for an image of Saturn from the nonrefurbished Hubble Telescope, preclassified into nine regions to obtain different degrees of smoothing in different regions. A Conditional Auto-Regressive (CAR) model has been used to incorporate smoothness constraints (Molina et al. 1994a). The CAR model used in astronomy is a Markovian Gaussian model. After a composition of the CAR prior model, the blurred image equation and the 
Poisson statistics in a bayesian framework, the estimation of maximum a posteriori (MAP) gives a smoothed RL restoration with a variable regularization factor for every step of restoration. That may be the reason that produces an oversmoothing for low values of SNR ratio (Molina 1994b). Here we present a new regularization method for RL restoration, named FPR. We begin with the classical RL algorithm and we make an extension directly in the iterative scheme with a constant regularization factor, avoiding thus the oversmoothing effects. Our method also preserves the local photometric properties.

\section{Recall of the Richardson-Lucy restoration algorithm}

We consider the case of ground-based optical or infrared imaging by a single aperture. Let $O(x, y)$ be the true twodimensional intensity distribution of the object, where $x, y$ are two orthogonal coordinates in some small region of the sky. The measured data $D(x, y)$ after "bias" and "flatfield" corrections takes the form of a convolution:

$$
\begin{aligned}
D(x, y) & =\iint H(x-u, y-v) O(u, v) \mathrm{d} u \mathrm{~d} v+N(x, y) \\
& =(H * O)(x, y)+N(x, y),
\end{aligned}
$$

where $*$ indicates two-dimensional convolution, $H(x, y)$ is the point spread function (PSF) of the imaging system and $N(x, y)$ represents the additive noise.

We shall from now on adopt a discrete representation of signals, i.e. work on a discrete two-dimensional lattice of sites, $S=\{s\}$. The true scene will then be noted as: $O=\left\{O_{s}\right\}_{s \in S}$ or equivalently: $O=O(i, j)$ with $(i, j) \in$ $\mathrm{Z}^{2}$. It yields a blurred image after discrete convolution as: $I=H * O$, where the PSF of the blur response can be written: $H=\left\{H_{s r}\right\}$ with $\left\{H_{s r}\right\}=H_{s r}=H_{r-s}$ for each pair of sites $(s, r) \in S$ (it corresponds to $H(x-u, y-v$ ) in Eq. (1)). Then the observed noisy data, $D=\left\{D_{s}\right\}_{s \in S}$, are obtained by applying a Poisson distribution to $I$. Thus the following scheme applies:

$$
O \underset{\text { blur }}{\rightarrow} I=H^{*} O \underset{\text { noise }}{\rightarrow} \quad D=\text { Poisson }\left(H^{*} O\right) .
$$

The foundation of RL is to maximize the likelihood of the original image $O$, which can be written as:

$$
\begin{aligned}
P(D / O) & =P(D / I=H * O) \\
& =\prod_{r \in S} \exp \left\{-\left(H * O_{r}\right)\right\} \frac{\left[(H * O)_{r}\right]^{D_{r}}}{D_{r} !} .
\end{aligned}
$$

The $\log$-likelihood $L=\log P(D / O)$, is easily written as:

$L=\sum_{r \in S}\left\{-(H * O)_{r}+D_{r} \log (H * O)_{r}-\log \left(D_{r} !\right)\right\}$,

where a typical convolution product in the previous formula expands as:

$(H * O)_{r}=\sum_{s \in S} H_{s r} O_{s}$.
The Maximum Likelihood (ML) principle allows us to find the set of variables $\left\{O_{s}\right\}$ verifying:

$$
\frac{\partial L}{\partial O_{s}}=-\sum_{r \in S} H_{s r}+\sum_{r \in S} D_{r} \frac{H_{s r}}{(H * O)_{r}}=0 \forall s \in S .
$$

Assuming that the point spread function $H$ is normalized, i.e.

$$
\sum_{s \in S} H_{s r}=1 \forall r \in S
$$

implies in turn:

$$
1=\sum_{r \in S} \frac{D_{r} H_{s r}}{(H * O)_{r}} \quad \forall s \in S .
$$

The Richardson-Lucy restoration method starts from this set of exact equations. Multiplying each of these equation for site $s$ by the related original data $O_{s}$ yields:

$O_{s}=\sum_{r \in S} \frac{D_{r} H_{s r} O_{s}}{(H * O)_{r}} \quad \forall s \in S$

which is written in an iterative form justified by the EM algorithm (Shepp \& Vardi 1982)

$O_{s}^{(n+1)}=\sum_{r \in S} \frac{D_{r} H_{s r} O_{s}^{(n)}}{(H * O)_{r}^{(n)}} \quad \forall s \in S$.

We write this updating formula more concisely as:

$O^{(n+1)}=O^{(n)}\left[\frac{D}{H * O^{(n)}}\right] * H^{\mathrm{T}} \quad \forall n \geq 1$

and initialization $O^{(1)}$, where $H^{\mathrm{T}}$ is the transpose of $H$. This iterative scheme will also be noted in the following as:

$O^{(n+1)}=\operatorname{RL}\left(O^{(n)}\right), n \geq 1$.

It is easy to show that it preserves the global photometry since:

$$
\begin{aligned}
\sum_{s \in S} O_{s}^{(n+1)} & =\sum_{s \in S} \sum_{r \in S} \frac{D_{r} H_{s r} O_{s}^{(n)}}{(H * O)_{r}^{(n)}} \\
& =\sum_{r \in S} \frac{D_{r}}{(H * O)_{r}^{(n)}} \sum_{s \in S} H_{s r} O_{s}^{(n)} \\
& =\sum_{r \in S} D_{r} .
\end{aligned}
$$

Usually $O^{(1)}$ is taken as a uniform flat image having the same total flux as observation $D$.

\section{Flux-Preserving Regularization (FPR) method for restoration}

The main problem with the RL algorithm is that in practice it doesn't converge to the global maximum because of the fact that we are dealing with an ill-posed problem and some a priori knowledge, not contained in the maximum 
likelihood model, is needed. Data instances that are not compatible with others can cause singularities in the restoration solution. So, a regularization method is needed to replace the ill-posed problem with a well-posed problem (Titterington 1985; Katsaggelos et al. 1991). The regularization approach overcomes this difficulty by choosing among the possible objects one "smooth" object that approximate the data. The basic underlying idea in most regularization approaches is the incorporation of a priori knowledge into the restoration.

Assume now that we modify the iterative scheme (Eq. (4)) in this sense:

$O^{(n+1)}=(1-\lambda) \operatorname{RL}\left(O^{(n)}\right)+\lambda T\left(O^{(n)}\right) \quad \forall n \geq 1$,

where $T()$ is some operator regularizing the pixel intensities, and $\lambda$ is some positive constant lying between 0 and 1. We shall note from now on the total flux of an image $O$ as:

$$
F(O)=\sum_{s \in S} O_{s}
$$

From what precedes, the total flux evolves as:

$$
F\left(O^{(n+1)}\right)=(1-\lambda) F(D)+\lambda F\left(T\left(O^{(n)}\right)\right)
$$

When the operator $T()$ is chosen so that it preserves the total flux i.e.

$$
F\left(T\left(O^{(n)}\right)\right)=F\left(T\left(O^{(n-1)}\right)\right)
$$

then iteratively:

$$
F\left(O^{(n+1)}\right)=F\left(O^{(n)}\right) \ldots=F\left(O^{(1)}\right)=F(D)
$$

i.e. total flux is preserved, provided that the initial guess $O^{(1)}$ has the same total flux as the observation $D$.

We can for example choose for the preserving total flux operator $T($ ) any convolution filter associated with a normalized matrix $R$, for example a Gaussian filter whose standard deviation describes its spatial extension, or more simply a nearest-neighbor average filter, as will be used in the next section. The FPR algorithm takes now the form:

$$
O^{(n+1)}=(1-\lambda) O^{(n)}\left[\frac{D}{H * O^{(n)}}\right] * H^{T}+\lambda R * O^{(n)}
$$

Thus at each step the current pixel intensity will depend in a regularizing manner on its neighboring ones, according to the magnitude of parameter $\lambda$ (for $\lambda=0$ the FPR gives back the RL algorithm). It is also obvious that positivity is preserved when $0 \leq \lambda \leq 1$.

We have been unable up to now to prove the convergence of this algorithm along the lines of Shepp \& Vardi (1982).

\section{Results}

\subsection{Simulated images}

In practice, we begin with an initial artificial Titan image with the same apparent size as our real Titan image (Fig. 1). The PSF used is the real PSF measured by the ADONIS system (Figs. 2, 3). Both images have a size $64 \times 64$. The initial image $O$ (Fig. 1 ) has been convolved by the normalized PSF (Fig. 3) and corrupted by Poisson noise. The degraded image (Fig. 4) has been restored by RL and FPR methods. For every step of restoration the relative error $(R E)$ at of step $n$ has been estimated by (Eq.(7))

$R E^{(n)}=\sqrt{\frac{\sum_{s \in S}\left|O_{s}^{(n)}-O_{s}\right|^{2}}{\sum_{s \in S}\left|O_{s}\right|^{2}}}$.

In images containing an extended object, such as a galaxy or in our case Titan, some steps after the optimum (minimum) $R E$ of the RL algorithm, the extended emission develops a speckled appearance. Astronomical images of extended objects seems to present a minimal noise amplification after 5-25 iterations, where the RL algorithm gives the best result. In its practical application, the $\mathrm{RL}$ algorithm has to be stopped at the minimum $R E$ that can be found only in simulated images.

We have to choose the range of regularization operator $T$ (), namely four or eight-connectivity. Four (or eight) connectivity means that every pixel is connected with its four (or eight) nearest neighbors. In the case of the small image of Titan, a four-connectivity is enough, so that we use the same filter matrix $R$ for both the real and synthetic image:

$R=\left[\begin{array}{ccc}0 & 0.25 & 0 \\ 0.25 & 0 & 0.25 \\ 0 & 0.25 & 0\end{array}\right]$

The parameter $\lambda$ is kept constant throughout all iterations and can be chosen as $0.05,0.2,0.35$ or 0.5 . The FPR method seems to converge for every $\lambda>0.03$ with the filter $R$ which we have chosen (Figs. 7, 8), but we do not have any analytical convergence proof at the moment. The accepted convergence criterion is:

$\frac{\sum_{s \in W}\left|O_{s}^{(n+1)}-O_{s}^{(n)}\right|}{|W|}<\epsilon$,

where $W$ is a window of size $|W|=25 \times 25$ containing the whole Titan region, and $\epsilon=0.001$. This criterion is achieved for the simulated Titan image after 75 (for $\lambda=$ 0.5 ), 81 (for $\lambda=0.35$ ), 105 (for $\lambda=0.2$ ) and 252 (for $\lambda=0.05)$ iterations and for the real Titan image after 85 (for $\lambda=0.5$ ), 94 (for $\lambda=0.35$ ), 120 (for $\lambda=0.2$ ) and 300 (for $\lambda=0.05$ ) iterations. 


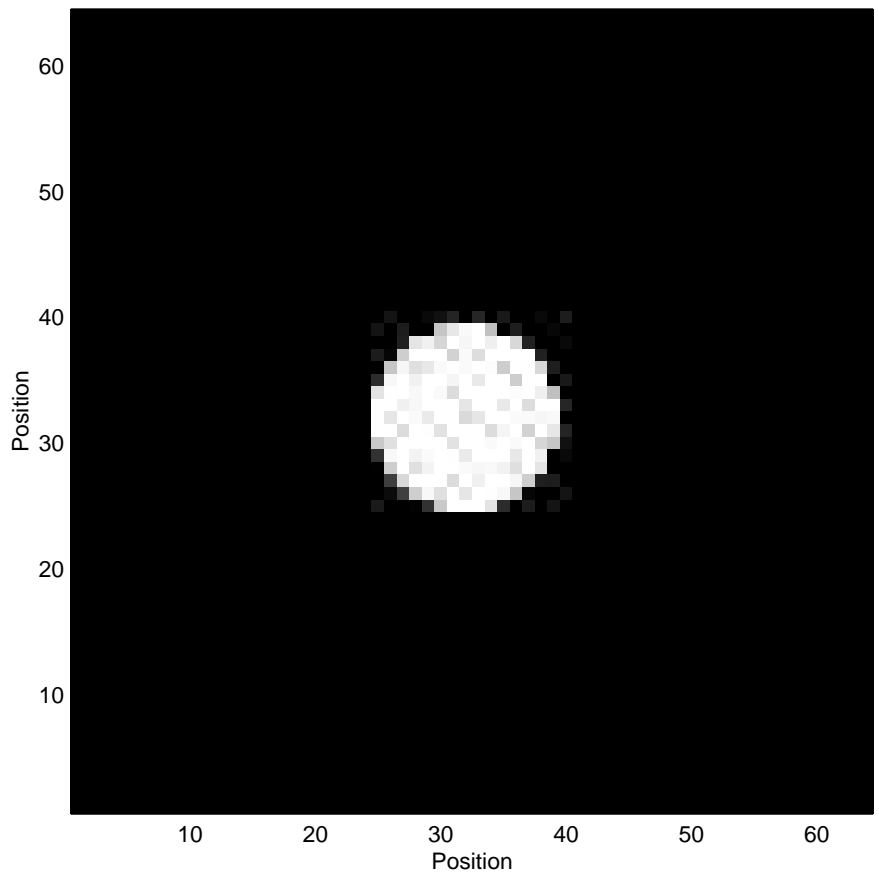

Fig. 1. Simulated image with the same apparent diameter of Titan.
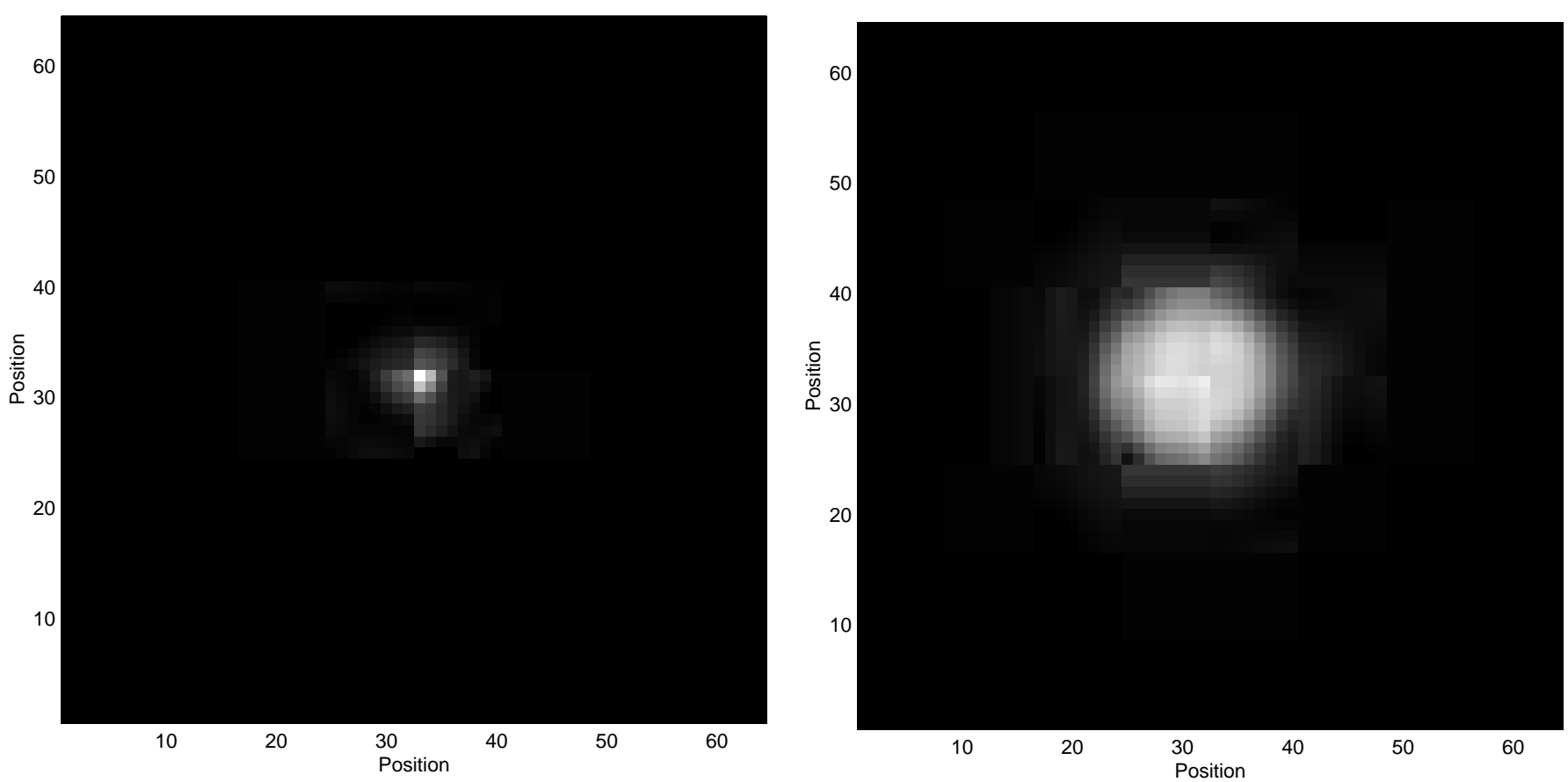

Fig. 2. PSF measured by ADONIS system.

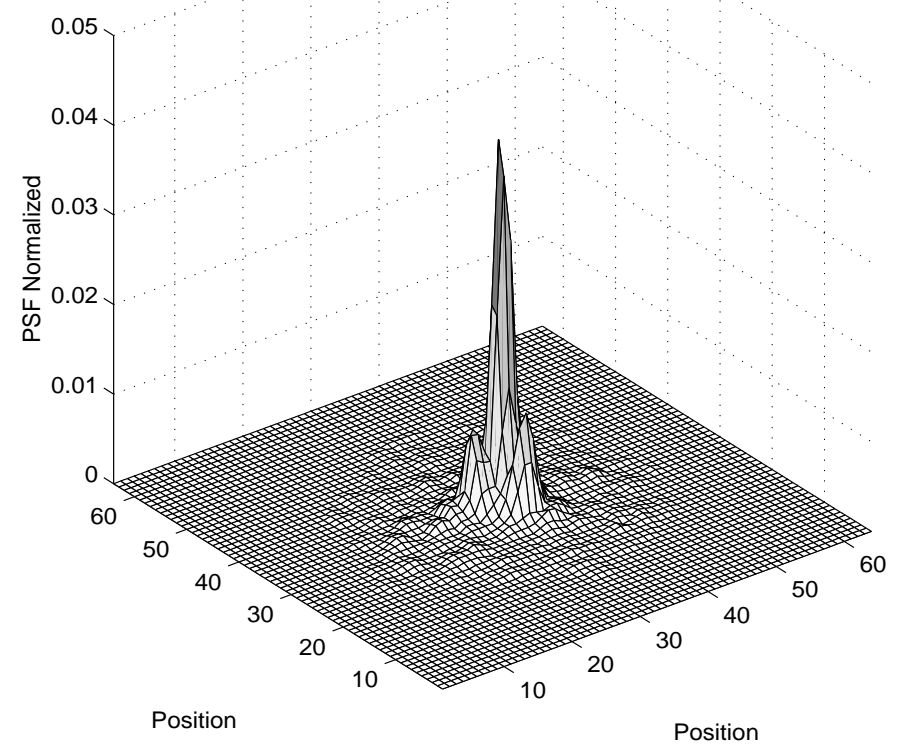

Fig. 3. PSF normalized to the sum of coefficients.

Fig. 4. Degraded simulated image of Titan. 


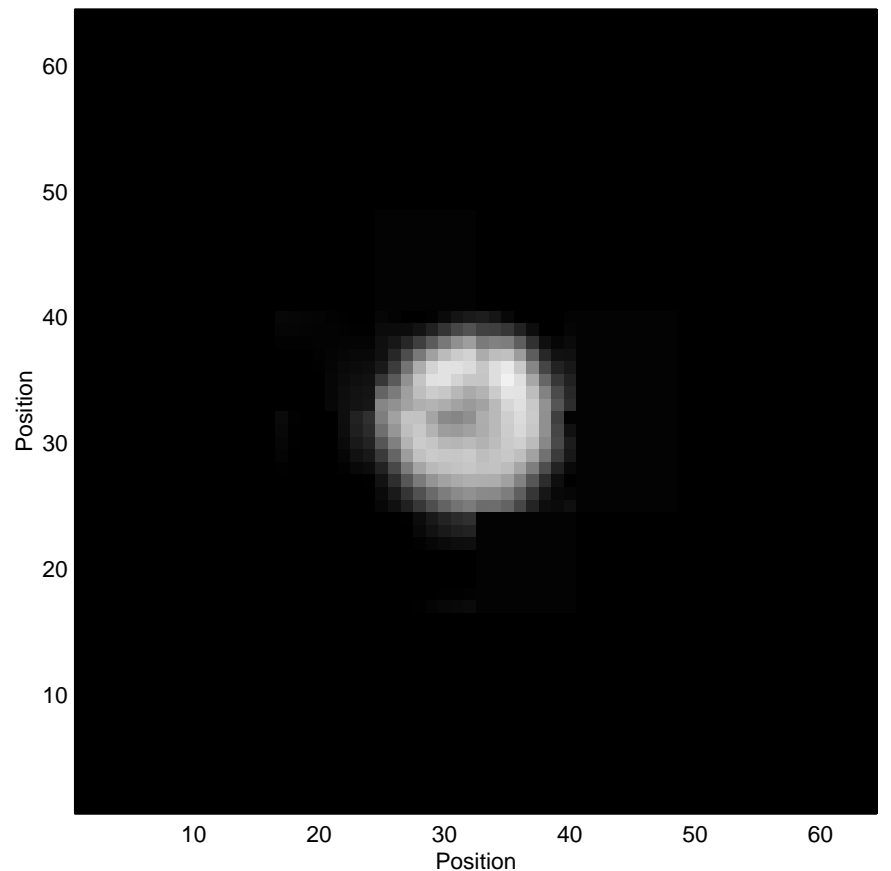

Fig. 5. Restored image with the optimum of RL method (after 25 steps).

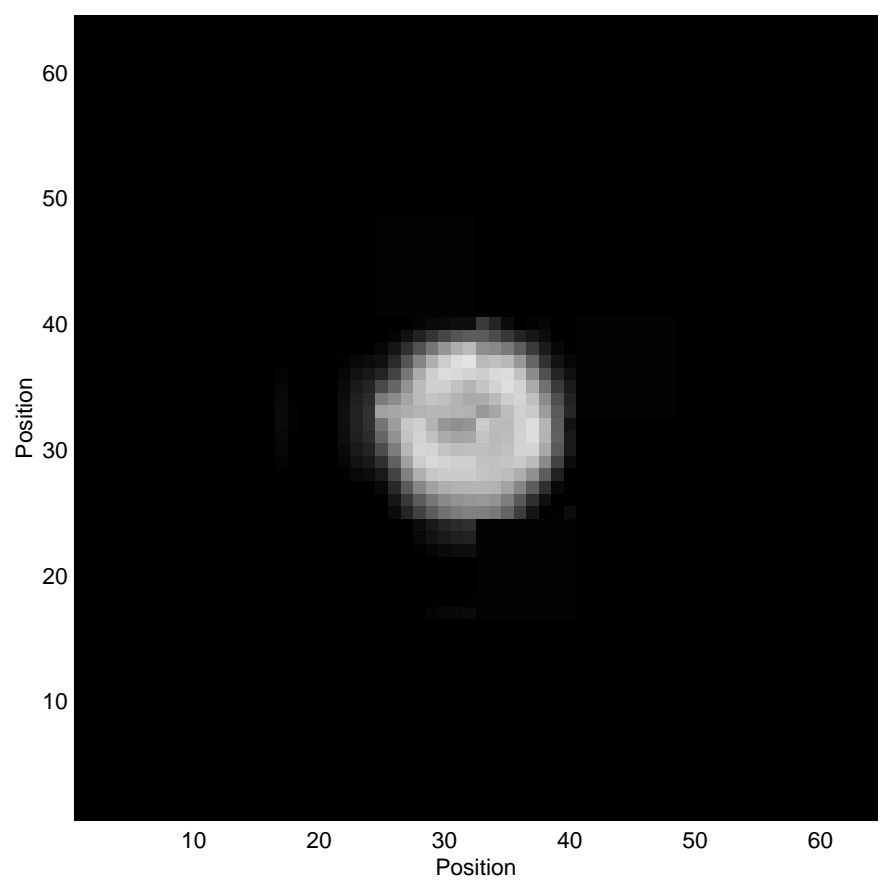

Fig. 6. Restored image with the FPR method and $\lambda=0.05$.

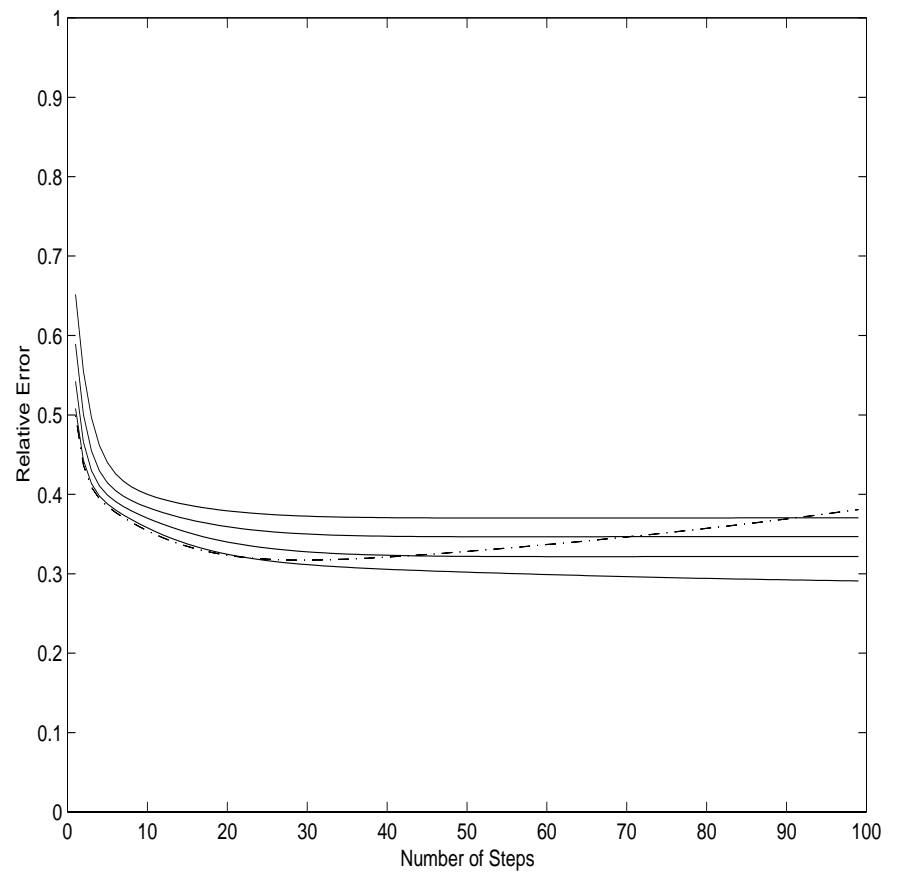

Fig. 7. The evolution of Relative Error after 100 steps. The dotted line presents the RL restoration and the solid lines the FPR restoration with $\lambda$ equal to $0.5,0.35,0.2$ and 0.05 from top to bottom.

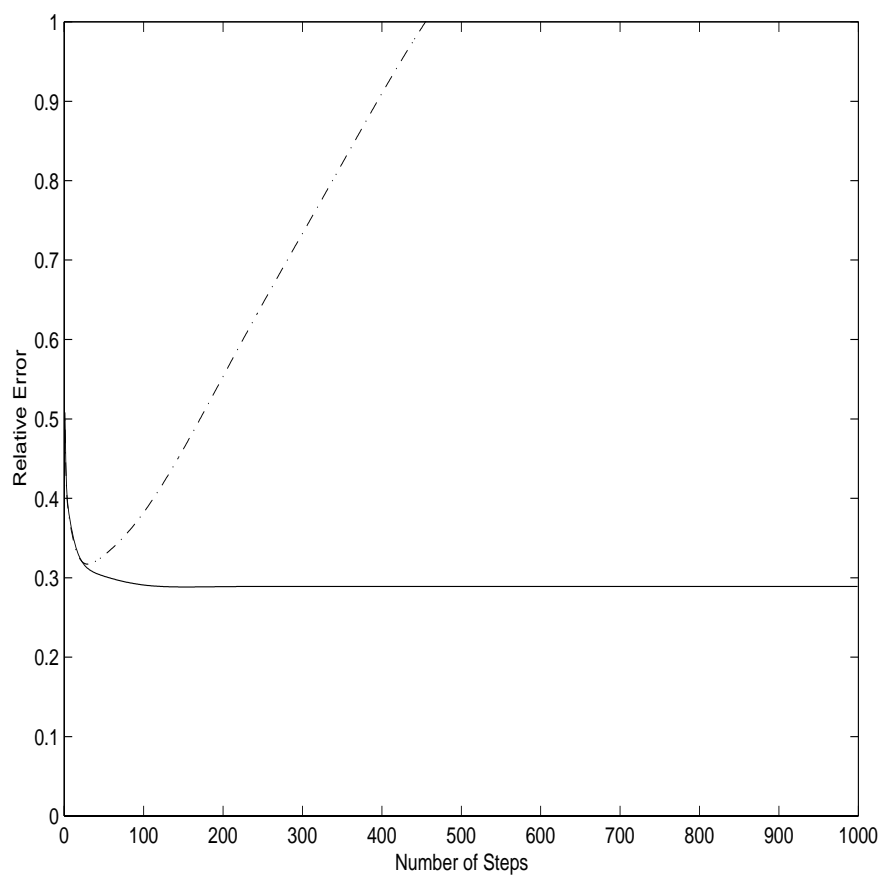

Fig. 8. The evolution of Relative Error after 1000 steps. The dotted line presents the RL restoration and the solid line the FPR restoration with $\lambda=0.05$. 


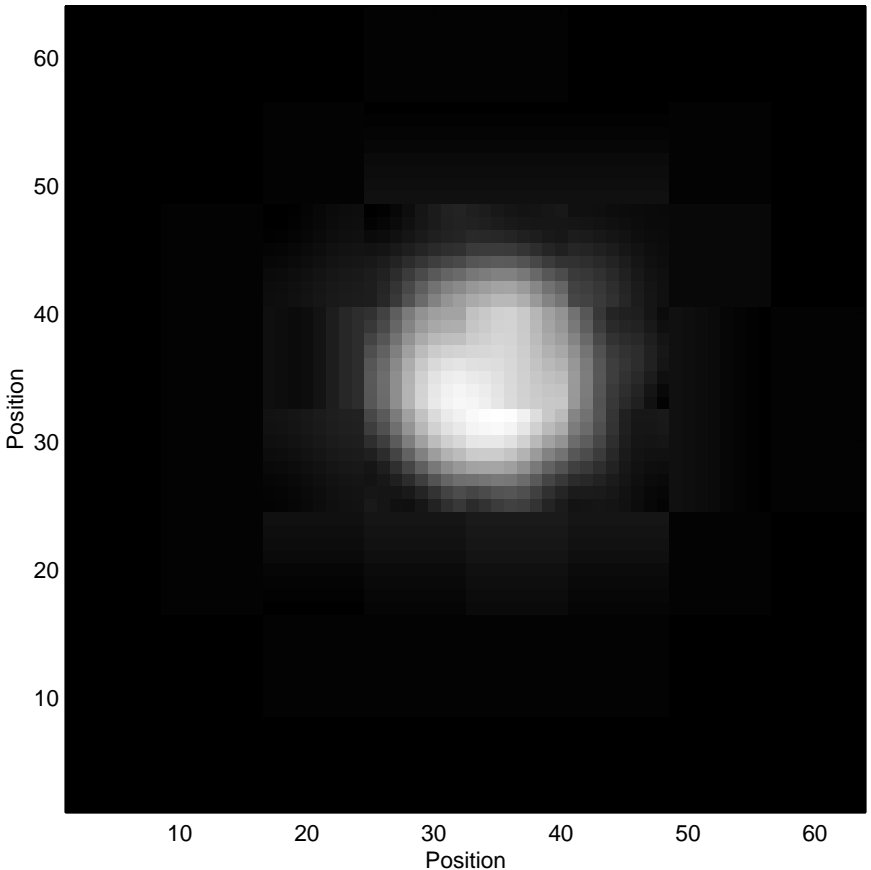

Fig. 9. Initial image of Titan $64 \times 64$.

\subsection{Results on real image}

The image of Titan (Fig. 9), $64 \times 64$ in pixel scale, was acquired with the adaptive optics system ADONIS installed at the ESO $3.6 \mathrm{~m}$ telescope in La Silla (Chile). The resolution is $0.05 \mathrm{arcsec} / \mathrm{pixel}$. The image was acquired at $2.04 \mu \mathrm{m}$, where the methane is transparent, with a narrowband filter, so it could be possible to see more details of surface. In adaptive optics compensation, the wavefront sensor data permit the estimation of the residual PSF. The PSF (Figs. 2, 3) was measured by the system two minutes before the image acquisition. The image has been corrected for systematic effects (Véran 1997; Combes et al. 1997). The region of interest is a sub-image containing the central region of Titan, with size $25 \times 25$. We can see the details in grey-scale contrast and with isophot contours in such sub-images for the initial observation and various restoration results (Figs. 10-21). We start with a flat image $O^{(1)}$ conserving the total flux of the observed image $D$, as explained in the previous section, and using the iterative method FPR (Eq. (6)) we obtain the following results (Figs. 14-21). These results can be compared with the RL restoration (Figs. 12, 13). Results for different values of $\lambda$ are given (Figs. 14-21). The isophot contours correspond to $0.98,0.95,0.9,0.85,0.82$ and 0.4 of the maximum intensity.

Figures 14 and 15 show the restoration results having the smallest relative error.

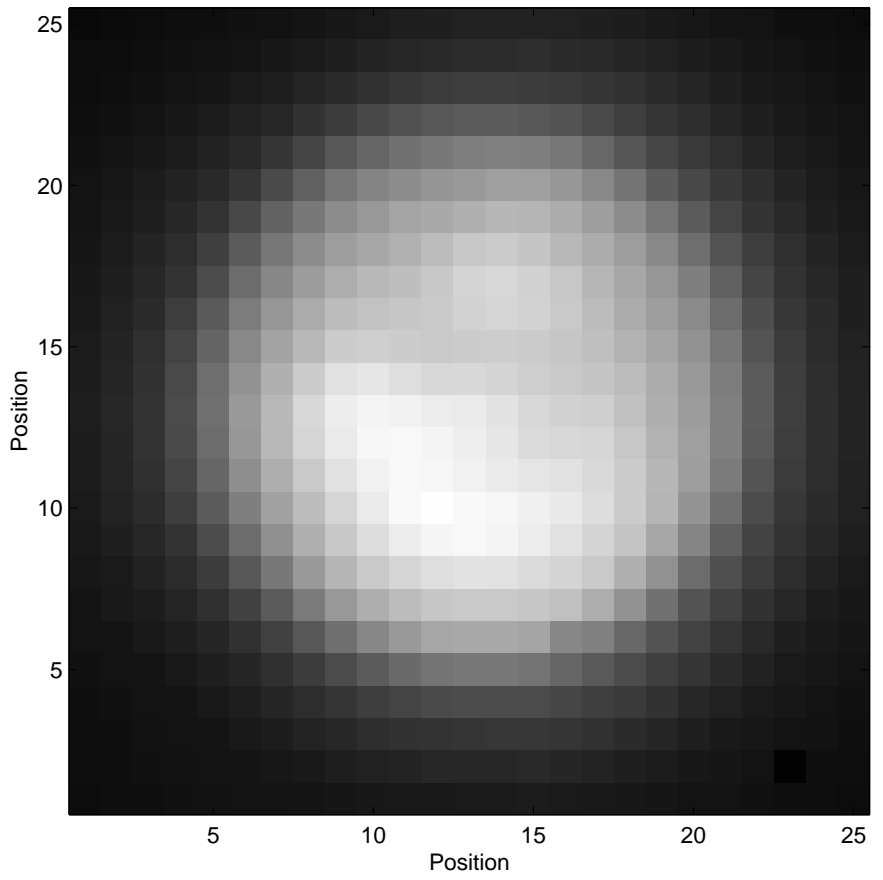

Fig. 10. Initial image of Titan $25 \times 25$.

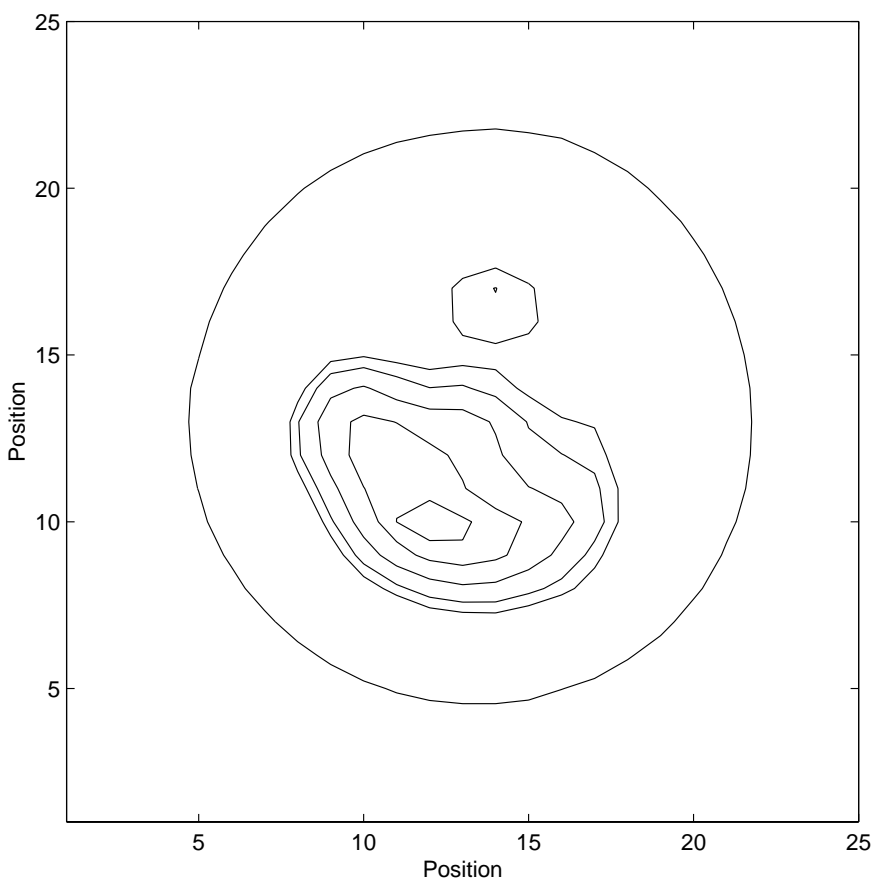

Fig. 11. Isophot contours corresponding to Fig. 10. 


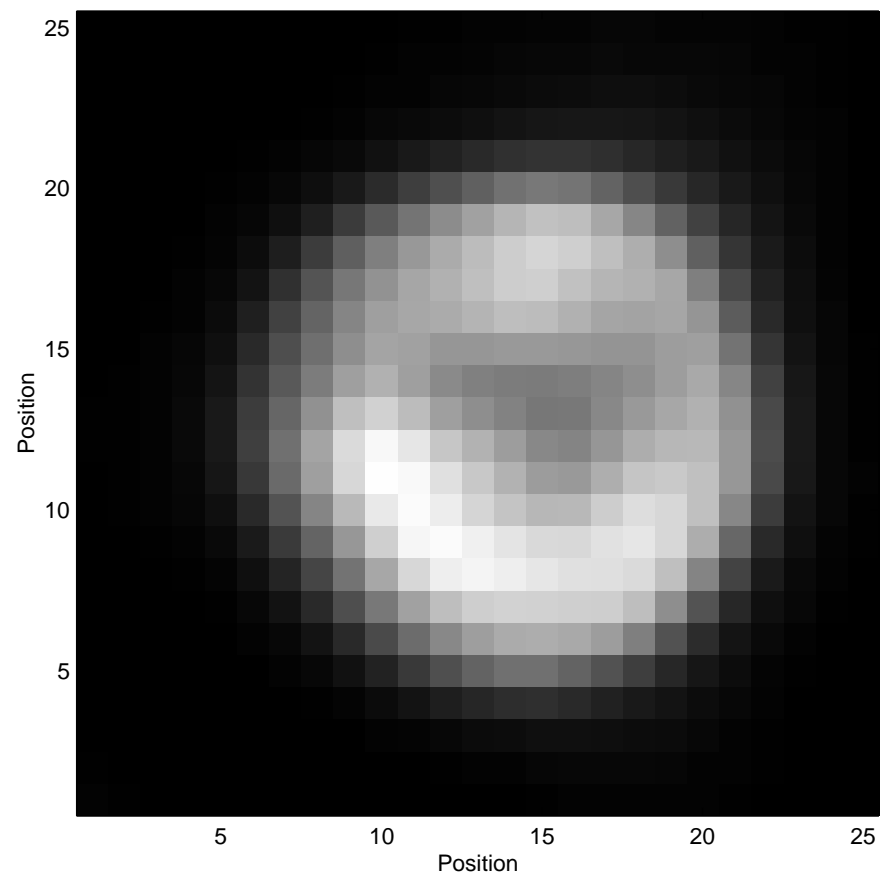

Fig. 12. Image of Titan after optimum RL restoration (25 steps).

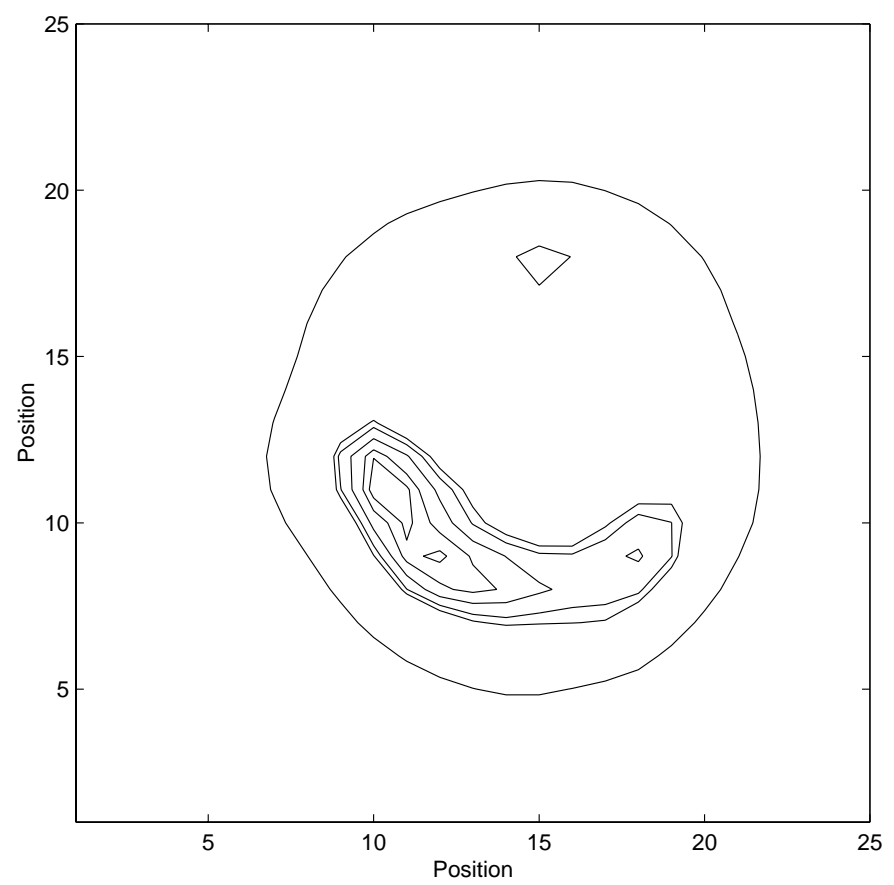

Fig. 13. Isophot contours corresponding to Fig. 12.

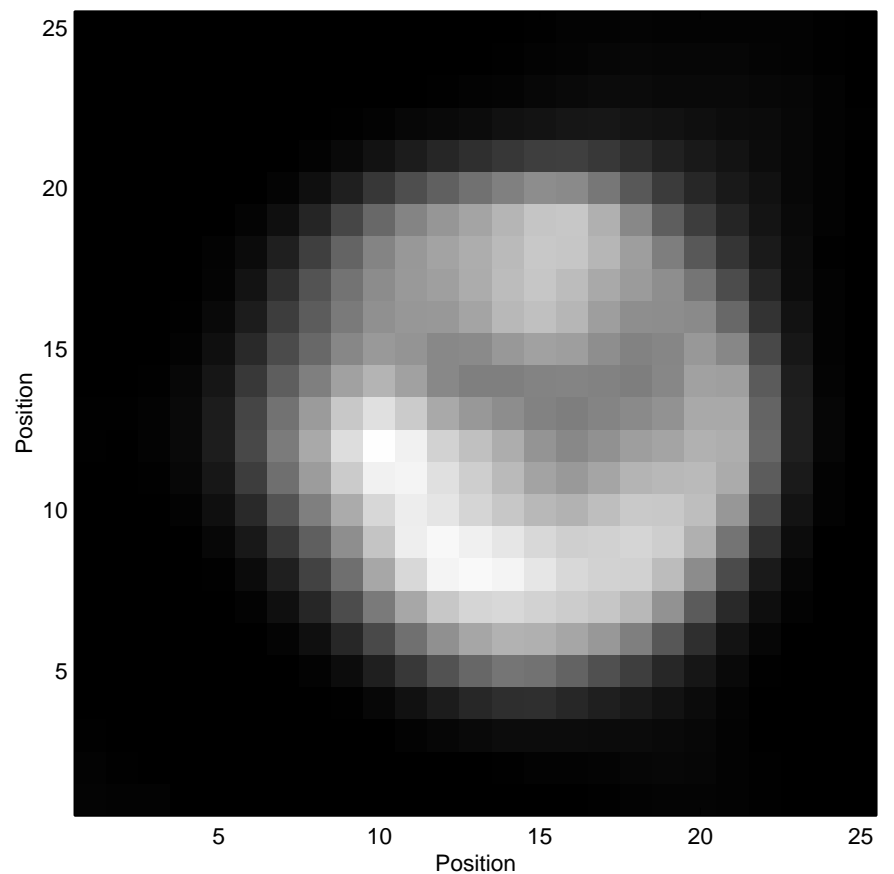

Fig. 14. Image of Titan after FPR restoration with $\lambda=0.05$.

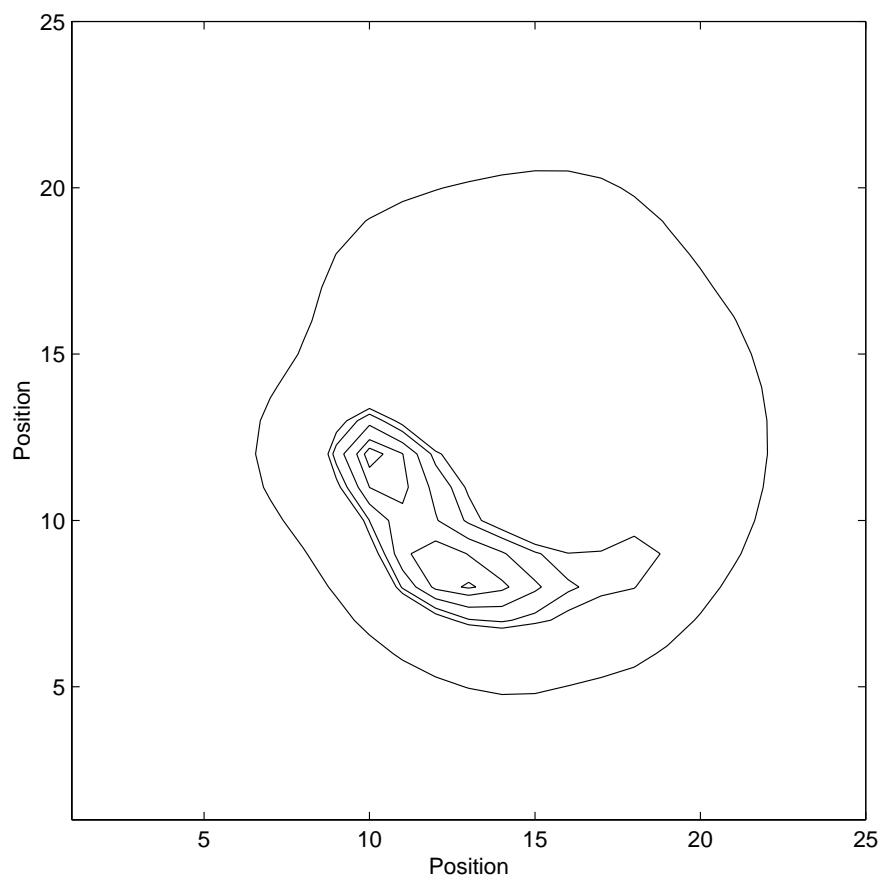

Fig. 15. Isophot contours corresponding to Fig. 14. 


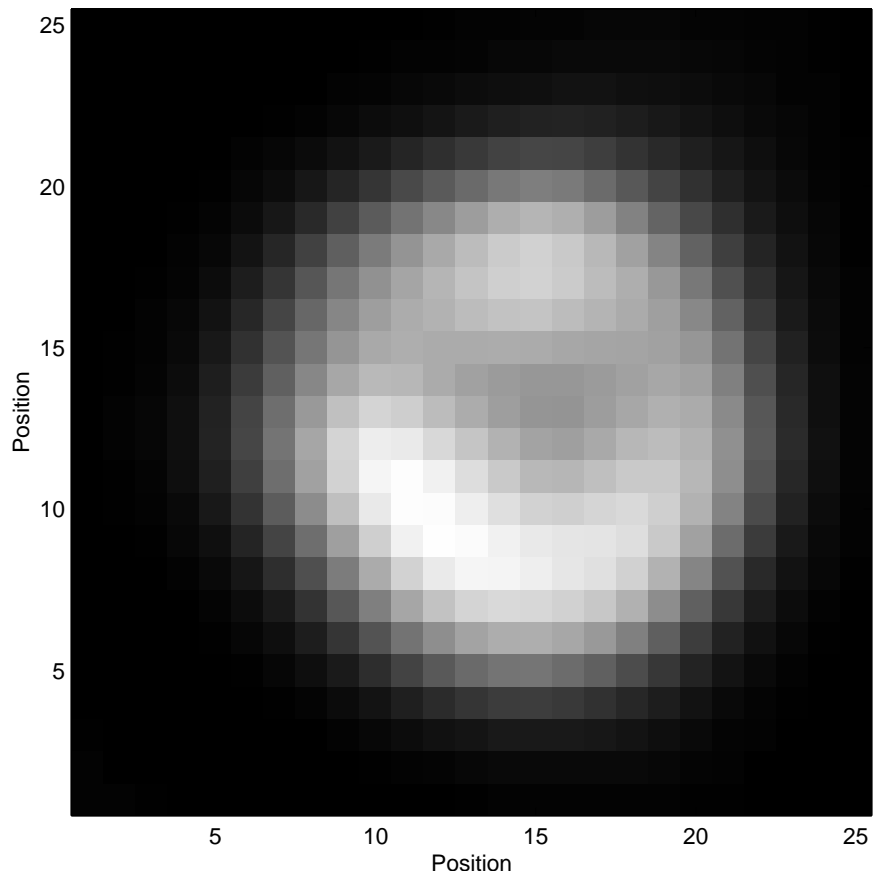

Fig. 16. Image of Titan after FPR restoration with $\lambda=0.2$.

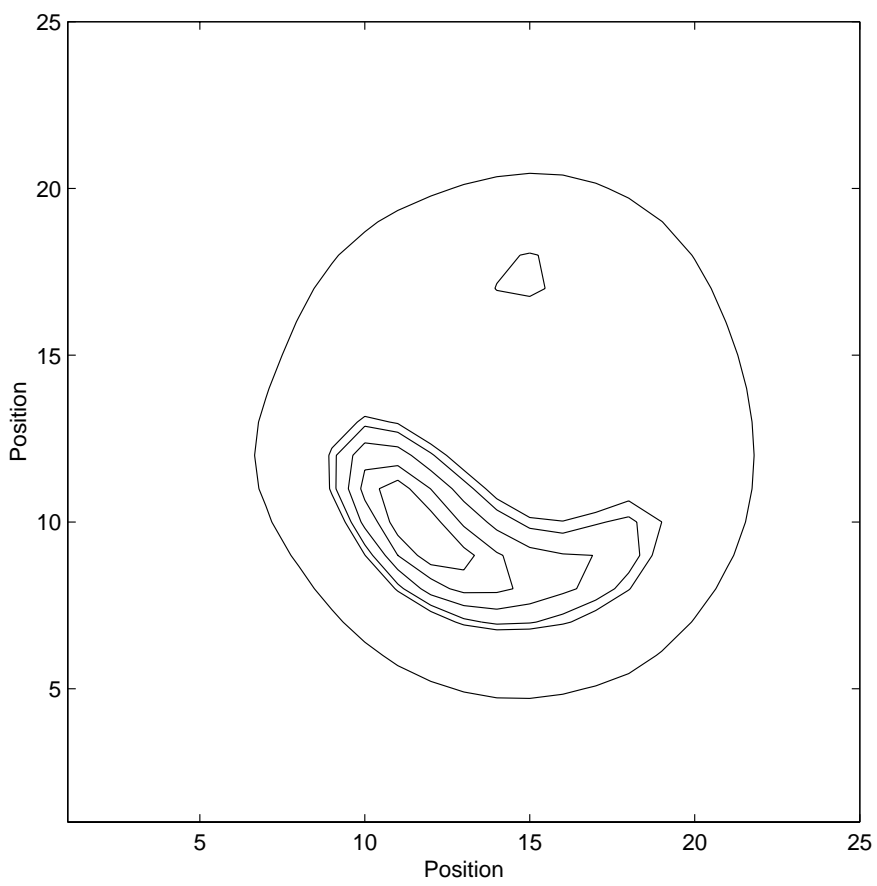

Fig. 17. Isophot contours corresponding to Fig. 16.

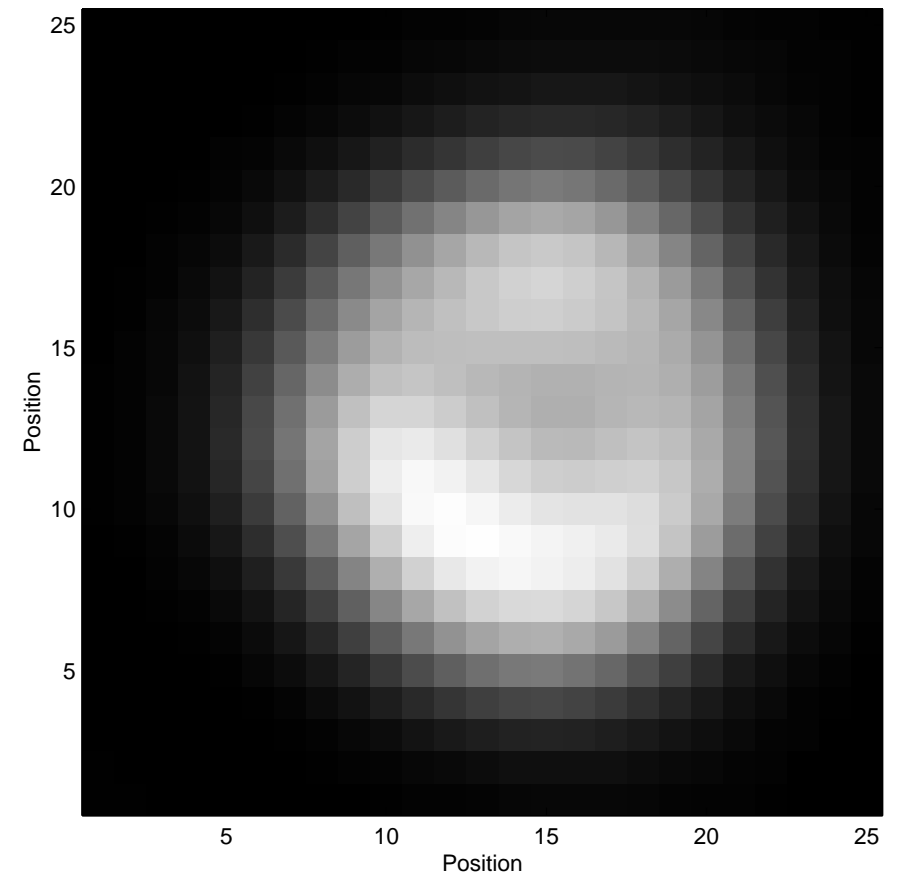

Fig. 18. Image of Titan after FPR restoration with $\lambda=0.35$.

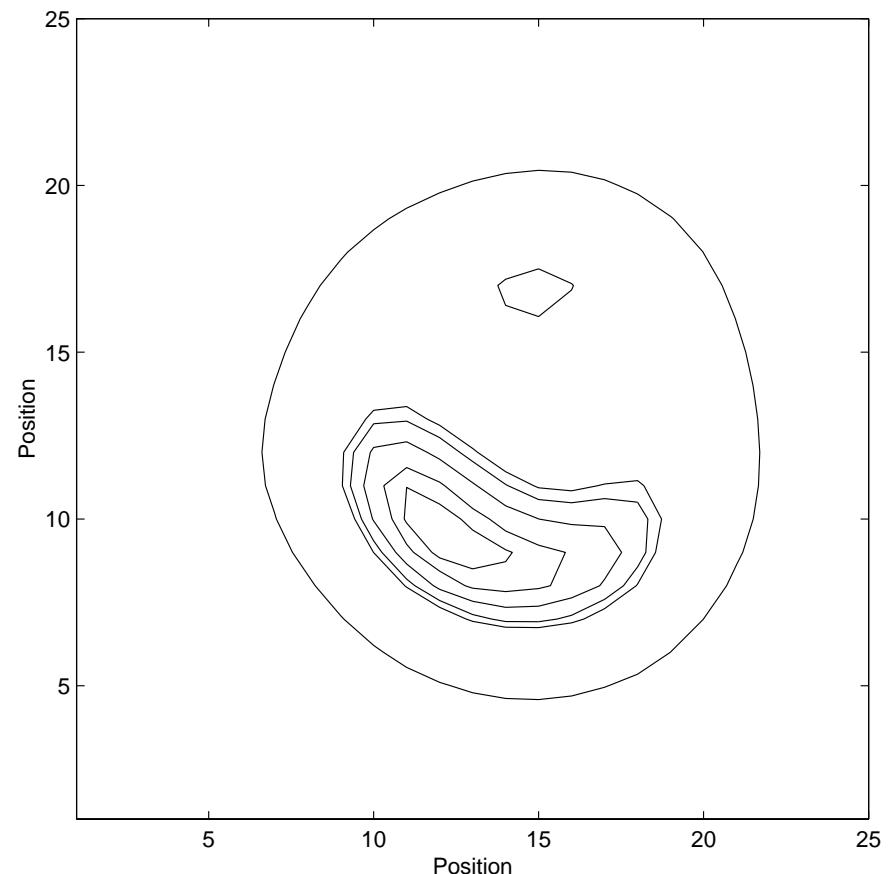

Fig. 19. Isophot contours corresponding to Fig. 18. 


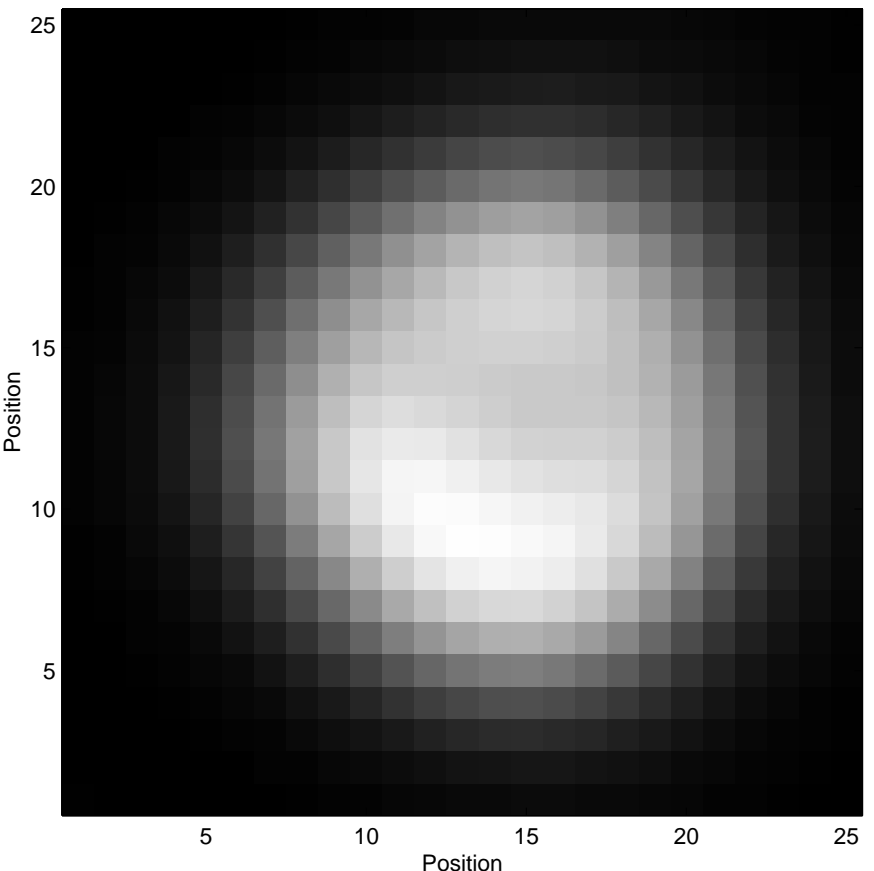

Fig. 20. Image of Titan after FPR restoration with $\lambda=0.5$.

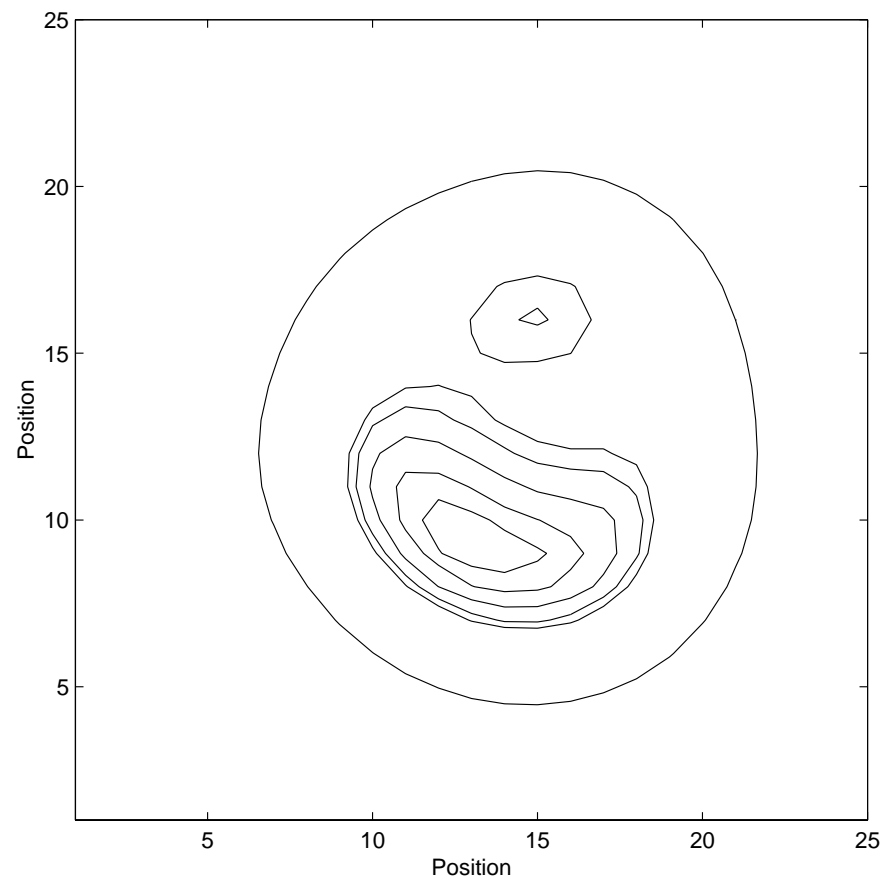

Fig. 21. Isophot contours corresponding to Fig. 20.

\section{Conclusions}

A new method, named FPR, has been proposed. The mathematical presentation has been presented as well as the results for a simulated image and for a real image of Titan acquired in near infrared with the adaptive optics system ADONIS. Comparing with the simulated tests, the best regularization parameter has been chosen as $\lambda=0.05$ (Fig. 7). In this case (Figs. 14, 15) the restored image presents two distinct sub-units and a possible deformation of Titan's surface.

Acknowledgements. The authors are grateful to J.-P. Véran, A. Coustenis, M. Combes and all the people who work for the adaptive optics system ADONIS for the loan of observational material.

\section{References}

Combes, M., Vapillon, L., Gendron, E., et al. 1997, Icarus, 129, 482

Hook, R. N., \& Lucy, L. B. 1994, in The Restoration of HST Images and Spectra II, ed. R. J. Hanish, \& R. L. White (STScI, Baltimore), 86

Hook R. N., \& Lucy, L. B. 1995, in Astronomical Data Analysis Software and Systems IV, ed. R. A. Shaw, H. E. Payne, \& J. J. E. Hayes, Ast. Soc. of the Pacific, 293

Katsaggelos, A. K., Biemond, J., Schafer, R. W., \& Mersereau R. M. 1991, IEEE Trans. Signal Proc., 39, 914

Lucy, L. B. 1974, AJ, 79, 745

Lucy, L. B. 1994, in The Restoration of HST Images and Spectra II, ed. R. J. Hanish, \& R. L. White (STScI, Baltimore), 79

Molina, R., Mateos, J., \& Abad, J. 1994a, in The restoration of HST Images and Spectra II, ed. R. J. Hanish \& R. L. White (STScI, Baltimore), 118

Molina, R. 1994b, IEEE Trans. Patt. Anal. Machine Intell., 16, 1122

Murtagh, F., Starck, J.-L., \& Bijaoui, A. 1995, A\&AS, 112, 179

Núñez, J., \& Llacer, J. 1998, A\&AS, 131, 167

Richardson, W. H. 1972, J. Opt. Soc. Amer., 62, 55

Shepp, L. A., \& Vardi, Y. 1982, IEEE Trans. Medical Imaging, 1,113

Snyder, D. L., Hammoud, A. M., \& White, R. L. 1993, J. Opt. Soc. Amer. A, 10, 1014

Snyder, D. L., Helstrom, C. W., Lanterman, A. D., Faisal, M., \& White, R. L. 1995, J. Opt. Soc. Amer. A, 12, 272

Starck, J.-L., Murtagh, F., \& Bijaoui, A. 1994, in The Restoration of HST Images and Spectra II, ed. R. J. Hanish, \& R. L. White (STScI, Baltimore), 111

Starck, J.-L., \& Murtagh, F. 1994, A\&A, 288, 342

Titterington, D. M. 1985, A\&A, 144, 381

Véran, J.-P. 1997, Ph.D. Thesis, École Nationale Supérieure des Télécommunications, ENST97E036, Paris 